\title{
Relative Entropy Sorting Method Based on the Preference Information of Alternative
}

\author{
Yang Yi \\ State Grid Henan Electric Power Company Information \\ \&Telecommunication Company \\ Zhengzhou,China \\ e-mail:deadfox@sina.com
}

\section{Lian Bizhan}

State Grid Henan Electric Power Company Information \&Telecommunication Company

Zhengzhou,China

e-mail: lianbizhan@ha.sgcc.com.cn

\author{
Wang Shiwen \\ State Grid Henan Electric Power Company Information \\ \&Telecommunication Company \\ Zhengzhou,China \\ e-mail: wangshiwen@ha.sgcc.com.cn \\ Gao Jianwei* \\ North China Electric Power University \\ School of Economics and Management \\ Beijing,China \\ e-mail: gaojianwei111@sina.com
}

\begin{abstract}
Multiple attribute decision making (MADM) problems are to find a desirable solution from a finite number of feasible alternatives assessed on multiple attributes, both quantitative and qualitative. In the recent years, MADM has received a great deal of attention from researchers. This paper studies the multi-attribute decisionmaking problem of power communication resources investment, where both the attributes values of the alternatives and the subjective preference information of the alternatives are interval values. We provide a decisionmaking approach based on the distance of relative entropy. In addition, for the attributes weights are completely unknown or partially known, we calculate them by establishing the optimization model based on the minimization deviation of subjective and objective information. The deviation between subjective assessment and objective information is determined based on the distance of relative entropy. Furthermore, the order of the solutions is determined by the relative entropy distances from objective and subjective preferences of each solution. Finally, an example is illustrated to examine the effective of our method.
\end{abstract}

Keywords-multiple attributes decision-making; interval value; relative entropy; attributes weights

\section{INTRODUCTION}

Currently, the multi-attribute decision making theory and methods based on the explicit program evaluation have been more perfect $[1,3]$. However, due to the complexity and the decision-makers limited cognition of objective things, the decisions made by the objective information alone will lead it not accurate. So multiple attribute decision making problem with subjective evaluation of program has attracted people's attention gradually [4,7]. Reference[4] is studied on multi-attribute decision making problems which attribute weights are completely unknown and preference information is provided in the form of interval number , and it proposes a decision-making method based on the deviation degree. Reference[5] is studied on multi-attribute decision making problems which attribute weights are known partially and preference information is provided in the form of interval number , and it proposes a decision-making method based on the objective programming model. Reference[6] is studied on multi-attribute decision making problems which attribute weights are known partially and preference information is provided in the form of complementary judgment matrix and reciprocal judgment matrix. Reference[7] is studied on multi-attribute decision making problems which attribute weights can not fully be ascertained, program property and preference information are provided in the form of triangular fuzzy number Reference[8] is studied on multi-attribute decision making problems which program property and preference information is provided in the form of intuitionistic fuzzy numbers. Reference[9] is studied on multi-attribute decision making problems which program property is provided in the form of interval number and preference information of the program is provided, and it proposes a decision-making method based on the grey relational analysis;

Entropy as the best measure of uncertainty is widely used in various disciplines. In recent years, the principle of entropy optimization is applied successfully in the multiattribute decision making problems [1], and it has achieved certain results. According to the relative entropy theory, reference[10] proposes a method to determine the weights of experts. According to the relative entropy theory, reference[11] proposes a combination weighting approach based on the multi-attribute decision making. According to the relative entropy which comes from the comparison between the evaluation solution, the ideal solution and negative ideal, reference[12] proposes a sort method of multi-attribute decision making. For multi-granularity uncertain linguistic and multi-attribute group decision making problems with incomplete policymakers attribute weight information, reference[13] proposes a possibledegree sort method based on the relative entropy. For the consistency problem of complementary judgment matrix, reference[14] proposes a relative entropy sorting method 
based multiplicative and additive. For uncertainty multiattribute decision making problems that decision matrix elements is the fuzzy values of three parameters interval values, reference[15] proposes a relative closeness sort method based on the relative entropy. On the basis of grey system, reference[16] proposes a relative entropy gathering model based on the gray relational analysis. For uncertain multiple attribute decision making problems that decision matrix element is interval number, reference[17] proposes a closeness sorting method based on relative entropy.

However, reference[17] does not consider the interval multi-attribute decision making problems with uncertain and subjective evaluation information about the program. For this problem, this paper presents an interval multiple attribute decision analysis method based on relative entropy. And on the cases of that the weight information is unknown completely or is know partially, the optimal model is build to solved the problems respectively. The method of this paper is without the comparison of interval numbers and the calculations are more simple. Finally, through the study of the case, the effectiveness of the proposed method is illustrated.

\section{PRELIMINARIES}

Definition 1: Set $R$ to real number, where $a=\left[a^{L}, a^{U}\right] a^{L} \leq a^{U}$ is known as the closed interval. In particular, when $a^{L}=a^{U}, a$ is degraded to the certain number.

Definition 2:According to the information theory, there are two systems $\mathrm{A}$ and $\mathrm{B}$ and the extent of the difference between their state $A_{i}$ and $B_{i}(\mathrm{i}=1,2, \cdots, \mathrm{n})$ is the available to measure by the Kullback-Leibler distance [18] .

$$
C_{i}=A_{i} \log \frac{A_{i}}{B_{i}}+\left(1-A_{i}\right) \log \frac{1-A_{i}}{1-B_{i}}
$$

The degree of difference between the two systems A and $\mathrm{B}$ is

$$
C=\sum_{i=1}^{n}\left\{A_{i} \log \frac{A_{i}}{B_{i}}+\left(1-A_{i}\right) \log \frac{1-A_{i}}{1-B_{i}}\right\}
$$

The objective preference value about attribute $G_{j}$ of program makers is $a_{i j}$ and the subjective preference value is $\alpha_{i}$.This paper definite the relative entropy distance of $a_{i j}$ and $\alpha_{i}$ as follows:

$$
d\left(a_{i j}, \alpha_{i}\right)=\sum_{i=L, R}\left[a_{i j}{ }^{i} \log \frac{a_{i j}{ }^{i}}{\alpha_{i}{ }^{i}}+\left(1-a_{i j}{ }^{i}\right) \log \frac{1-a_{i j}{ }^{i}}{1-\alpha_{i}{ }^{i}}\right]
$$

In the actual decision-making process, in order to make the decision more accurate and reasonable, it should make the differences between the subjective preference of the program and objective preference for the various attributes the smallest.

\section{POWER COMMUNICATION RESOURCE INVESTMENT DECISION PROBLEM CONSIDERING THE SUBJECTIVE INTERVAL EVALUATION}

Power communication resource investment scale is big and involved with complicated related factors,so it is difficult to accurately estimate the cost to the investment schemes and possible benefits. In reality, it generally adopts the method of combining the expert group evaluation and the financial accounting, so the scheme evaluation value is usually a interval data.Here are norms expressing such problems.

Considering $\mathrm{m}$ feasible investment program $A_{1}$, $A_{2}, \ldots, A_{m}, \mathrm{n}$ valuation attributes $G_{1}, G_{2}, \ldots, G_{n}$.The subjective evaluation information of experts to plan $A_{i}$ is given in the form of interval numbers,as $\alpha_{i}=$ $\left[\alpha_{i}^{L}, \alpha_{i}^{R}\right], \mathrm{i}=1,2, \ldots, \mathrm{m}$. The attribute value of plan $A_{i}$ under the valuation attribute $G_{j}$ is interval number $\left[x_{i j}^{L}, x_{i j}^{R}\right]$ and the decision matrix is $A$.Try to synthese the evaluations of subjective and objective to determined the optimal investment program.

$$
A=\left(\begin{array}{ccc}
{\left[x_{11}^{L}, x_{11}^{R}\right]} & \mathrm{K} & {\left[x_{1 n}^{L}, x_{1 n}^{R}\right]} \\
\mathrm{M} & \mathrm{O} & \mathrm{M} \\
{\left[x_{m 1}^{L}, x_{m 1}^{R}\right]} & \mathrm{L} & {\left[x_{m n}^{L}, x_{m n}^{R}\right]}
\end{array}\right)
$$

IV. POWER COMMUNICATION RESOURCE INVESTMENT DECISION METHOD BASED ON RELATIVE ENTROPY SORTING

For those power communication resource investment problems considering subjective evaluation, this paper uses the difference between the subjective information and objective evaluation information of investment program, which is based on a model constructed by relative entropy distance, and presents constrained nonlinear programming model, of which attribute weights are determined, goal is minimizing the difference between subjective and objective and the known part weight information is constraint. The decision-making process steps are as follows:

Step1. Standardization process of interval decision matrix should be conducted. Then the normalization matrix is denoted $\mathrm{Y}=\left(\left[y_{i j}^{L}, y_{i j}^{R}\right]\right) m \times n$.

Step2. Build a linear programming model to solve the optimal attribute weights. In the actual decision-making process, due to the complexity of objectives and the limitations of policy makers, clear attribute weight information is difficult to be determined, there will be a situation of attribute weight information is incomplete, even completely unknown. For this type of decisionmaking problems, it is necessary to give a reasonable method to determine the weight. To this end, this paper 
provides an evaluation difference minimization model based on relative entropy.

A. If the attribute weights partially known, there are six cases[19.20]:

$$
\begin{aligned}
& \bar{w}_{i} \geq w_{j} ; \alpha w_{i}-w_{j} \geq \alpha ; \beta w_{i} \geq \beta w_{j} ; \\
& \chi \gamma_{i} \leq w_{i} \leq \gamma_{i}+\varepsilon_{i} ; \\
& \delta \theta_{i} w_{j} \leq\left(\theta_{i}+\varepsilon_{i}\right) w_{i} \text { or } \theta_{i} \leq \frac{w_{i}}{w_{j}}\left(\theta_{i}+\varepsilon_{i}\right), w_{j} \neq 0 ; \\
& \varepsilon w_{i}-w_{j} \geq w_{k}-w_{l} ; \quad j \neq k \neq l 。
\end{aligned}
$$

Here, $\alpha_{i}, \beta_{i}, \gamma_{i}, \theta_{i}, \varepsilon_{i}$ are non-negative constants 。 Thus it can establish the following single objective optimization model :

$$
\begin{gathered}
\min D(w)=\sum_{i=1}^{m} \sum_{j=1}^{n} d\left(y_{i j}, \alpha_{i}\right) w_{j} \\
\text { s.t.w } \in W, \sum_{j=1}^{n} w_{j}=1, w_{j} \geq 0, j=1, \ldots, n \\
d\left(y_{i j}, \alpha_{i}\right)=\sum_{i=L, R}\left[y_{i j}{ }^{i} \log \frac{y_{i j}{ }^{i}}{\alpha_{i}{ }^{i}}+\left(1-y_{i j}{ }^{i}\right) \log \frac{1-y_{i j}{ }^{i}}{1-\alpha_{i}{ }^{i}}\right]
\end{gathered}
$$

presents the relative entropy distance of $a_{i j}$ and $\alpha_{i}$ as follows, where $a_{i j}$ is the objective preference value about attribute $G_{j}$ of program makers and $\alpha_{i}$ is the subjective preference value.$W$ represents some of the attributes of known weight information

$B$. If the attribute weights is completely unknown, we can establish the following single objective optimization model .

$$
\begin{aligned}
& \min D(w)=\sum_{i=1}^{m} \sum_{j=1}^{n} d\left(y_{i j}, \alpha_{i}\right) w_{j} \\
& \text { s.t. } \sum_{j=1}^{n} w_{j}{ }^{2}=1, w_{j} \geq 0, j=1, \ldots, n
\end{aligned}
$$

Solve the single objective optimization model, make it normalized to obtain optimal attribute weight vector $w_{j}$ :

$$
w_{j}=\frac{\sum_{i=1}^{n} d\left(y_{i j}, \alpha_{i}\right)}{\sum_{j=1}^{n} \sum_{i=1}^{n} d\left(y_{i j}, \alpha_{i}\right)}, j=1,2, \mathrm{~L}, n
$$

Step3. The relative entropy distances from objective and subjective preferences of each solution is calculated as follows.

$$
d_{i}=\sum_{j=1}^{n} d\left(y_{i j}, \alpha_{i}\right) w_{j}, i=1, \ldots, m
$$

Where $d_{i}$ sums up the overall relative entropy distances between objective and subjective preferences given by the decision makers of all characteristic from solution $A_{i}$.

Step4. Solution $A_{i}$ is ordered by the size of $d_{i}$. If $d_{i}$ is much bigger, it means that the objective preference given by decision makers is much closer to the subjective preference. Accordingly, the corresponding solution is much superior.

\section{CASE STUDY}

As for communication resource government, Electric Power Company of Henan needs to consider huge data and a variety of resources such as transmission network, switched network, service network and access network and so on. In order to advance the date processing and the efficiency, the company should contrast the five alternative power communication investment resource $A_{i}(\mathrm{i}=1, \ldots$, 5)and pick out the best one. What we will integrate the varieties of resources into are safety efficacy $G_{1}$, economic efficacy $G_{2}$ and manage efficacy $G_{3}$, according to which we evaluate investment cases. For each scheme, investor's subjective preference value is $\alpha_{1}=[0.30,0.70]$, $\alpha_{2}=[0.20,0.90], \quad \alpha_{3}=[0.10,0.45], \quad \alpha_{4}=[0.25$, $0.55], \alpha_{5}=[0.20,0.80] . \mathrm{Y}$ for the standardization of decision matrix, specific conditions as follows:

$$
Y=\left(\begin{array}{ccc}
{[0.214,0.220]} & {[0.166,0.178]} & {[0.184,0.190]} \\
{[0.206,0.225]} & {[0.220,0.229]} & {[0.182,0.191]} \\
{[0.195,0.204]} & {[0.192,0.198]} & {[0.220 .0 .231]} \\
{[0.181,0.190]} & {[0.195,0.205]} & {[0.185,0.195]} \\
{[0.175,0.184]} & {[0.193,0.201]} & {[0.201,0.211]}
\end{array}\right)
$$

Based on the method to determine the optimal scheme of Calculating steps are as follows:

$A$. If the attribute weight is partially known, assume that the attribute weight information is interval number, then $\mathrm{W}$ is :

$$
\begin{aligned}
& w_{1}=[0.3350,0.3755], \quad w_{2}=[0.3009,0.3138] \text {, } \\
& w_{3}=\left[\begin{array}{ll}
0.3194,0.3363
\end{array}\right]
\end{aligned}
$$

By formula (1) to establish the following linear programming problem:

$$
\begin{aligned}
& \min D(w)=1.3570 w_{1}+1.3728 w_{2}+1.4043 w_{3} \\
& \text { s.t. } w \in W, \sum_{j=1}^{n} w_{j}=1, w_{j} \geq 0, j=1,2,3 .
\end{aligned}
$$

get the optimal weight vector : 
$w=(0.3755,0.3051,0.3194)$. With the formula (4) calculate the relative entropy distance both objective and subjective preferences.

$$
\begin{gathered}
d_{1}=0.2490, \quad d_{2}=0.5689, \quad d_{3}=0.0748, \quad d_{4}=0.1198, \\
d_{5}=0.3644 .
\end{gathered}
$$

According to the value of solution to rank, $d_{3}<d_{4}<d_{1}<d_{5}<d_{2}$, among this five investment programmers, $A_{3}>A_{4}>A_{1}>A_{5}>A_{2}$.we can know the result $A_{3}$ is optimal.

$B$. If the attribute weight is completely unknown, you can use Formula (3) to gain the most optimal weight vector $w=(0.3282,0.3321,0.3397)^{\mathrm{T}}$, then use formula (4) to calculate the relative entropy distance between objective and subjective preferences.

$$
\begin{gathered}
d_{1}=0.2512, \quad d_{2}=0.5698, \quad d_{3}=0.0748, \quad d_{4}=0.1193, \\
d_{5}=0.3631
\end{gathered}
$$

According to $d_{i}$ to rank, $d_{3}<d_{4}<d_{1}<d_{5}<d_{2}$, therefore, among this five investment programmers, the result is $A_{3}>A_{4}>A_{1}>A_{5}>A_{2}$. So, scheme $A_{3}$ is optimal.

\section{CONCLUSIONS}

This paper studies the multi-criteria decision-making problem of power communication resources investment, where both the attributes values of the alternatives and the subjective preference information of the alternatives are interval values. We provide a decision-making approach based on the distance of relative entropy and use single objective optimization model to determine the attribute weights, without comparing the size of the interval number. In this way, the calculation is simpler. In addition, with due consideration of objective information and policy makers subjective intention, we can make the decisionmaking process more reasonable.

\section{ACKNOWLEDGMENT}

The authors are very grateful to the anonymous referees for their insightful and constructive comments and suggestions which have helped to improve the paper. This research is supported by the Natural Science Foundation of China under Grant No. 71271083.

\section{REFERENCES}

[1] Qiu Wanhua. Management decision-making and application of entropy [M].Beijing:Mechanical industry press ,2001.
[2] Xu Zeshui. The uncertain multi-attribute decision making method and application $[\mathrm{M}]$.Beijing:Tsinghua university press ,2004: 115131.

[3] Hang C L, Yoon K. Multiple Attribute Decision Making[M].Berlin: Spring-Verlag, 1981: 110-145.

[4] Xu Zeshui. New method for uncertain multi-attribute decision making problems[J].Journal of systems engineering,2002,17(2): 177-181.

[5] Jiang Yanping, Fan Zhiping. Method for multiple attribute decision making with attribute interval numbers and preference information on alternatives[J].Systems Engineering and Electronics,2005, 27(9):250 -252.

[6] Xu Zeshui. Method for multi-attribute decision making with preference information on alternatives under partial weight information[J]. Control and Decision, 2004,19(1): 85-88

[7] Liu Wei-feng, He Xia. A Triangular Fuzzy Number Multi-attribute Decision-making Method with Preference Information on Alternatives[J]. Fuzzy Systems and Mathematics,2013, 27(2): 155161.

[8] Wei Guiwu, Wei Yu, Model of Grey Relational Analysis for Interval Multiple Attribute Decision Making with Preference Information on Alternatives[J]. Chinese Journal of Management Science, 2008,16(1): 158-161

[9] He Xia, Liu Weifeng. An Intuitionistic Fuzzy Multi-attribute Decision-making Method with Preference on Alternatives $[\mathrm{J}]$. Operations research and management science ,2013,2(22): 36-40.

[10] Wan S P. Congregation of the experts' weights based on relative Entropy for group decision-making problem with incomplete information[J]. Communication on Applied Mathematics and Computation,2009,23(1): 66-70.)

[11] Zhou Yufeng, Wei Fajie. CombinationWeighting Approach in Multiple Attribute Decision Making Based on Relative Entropy[J]. Operations research and management science, 2006, 15(5): 48-53.

[12] Zhao Meng, Qiu Wanhua, Liu Beishang. Relative entropy evaluation method for multiple attribute decision making[J].Control and Decision,2010,25(7):1098-1104.

[13] Zhang Zhen, Guo Chonghui. A multi-granularity uncertain linguistic group decision-making method based on relative entropy[J].Journal of Dalian University of Technology,2012, 6(52):921-927.

[14] Lv Yuejin, Cheng Hongtao, Qin Juying. Complementary judgement matrix ranking method based on relative entropy[J].Systems Engineering-Theory\&Practice, 2011，31(7): 1328-1333.

[15] Zhao Meng, Qiu Wanhua. Interval-valued fuzzy multi-attribute decision making method based on relative entropy of three parameters [J].Statistics and Decision ,2012,354(6):58-61.

[16] Zhang Yanfeng.An Effective REM Assembly Based on the Grey Correlation Analysis[J]. Journal of Northwestern Polytechnical University.2013,31(2):255-258.

[17] Zhao Meng, Qiu Wanhua. He Dayi. Compositor Method of Relative Entropy for Interval Multi-attribute Decisionmaking[J].Systems Engineering,2010,28(8):70-74.

[18] Cover T M,Thomas Joy A. Elements of information theory [M] New York: John Wiley and Sons, 2006.

[19] Lee K S, Park K S, Eum Y S, et al. Extended methods for identifying dominance and potential optimality in multi-criteria analysis with imprecise information[J]. European Journal of Operational Research, 2001, 134(3):557-563.

[20] Kim S H, Han C H. An interactive procedure for multiple attribute group decision making procedure with incomplete information [J] Computers\& Operational Research,1999, 26(8):755-772. 